\title{
TOPOLOGICAL PROPERTIES OF SUBANALYTIC SETS
}

\author{
BY
}

\author{
ROBERT M. HARDT( $\left.{ }^{1}\right)$
}

\begin{abstract}
The stratification of a semianalytic or subanalytic set (that is, a set which locally is the proper analytic image of some semianalytic set) leads easily, by consecutive projections in Euclidean space, to a CW decomposition. In the category of subanalytic sets and continuous maps with subanalytic graphs, theories of slicing, intersection, and homology result through use of the topological chains defined by subanalytic sets.
\end{abstract}

1. Introduction. It has long been understood how semianalytic sets, which are sets locally defined by finitely many real analytic equalities and inequalities, admit stratifications into semianalytic, real analytic submanifolds of various dimensions [11, pp. 150-153]. The harder question of their triangulability was, after a somewhat stormy history, established in [3]. (See also [10].) A major problem was that the image of a semianalytic set under even a proper analytic map need not be semianalytic [11, p. 133]. Recently it was shown independently in [9] (first) and in [7] that such images, called subanalytic sets in [9] and semianalytic shadows in [7], admit stratifications into subanalytic, real analytic submanifolds. Our main goal here is to show how, for locally finite families of semianalytic (respectively, subanalytic) sets, these stratifications lead directly to certain semianalytic (resp., subanalytic) CW decompositions (3.1-3.3). For the semianalytic case locally, some aspects of this construction were first treated in [8, Lemma 3]. Triangulations by subanalytic maps have recently been found in [13].

A subanalytic map is a continuous function between subsets of real analytic manifolds whose graph is a subanalytic subset of the Cartesian product of the manifolds; a $k$ dimensional subanalytic chain is a sum of integral multiples of oriented $k$ dimensional members of some subanalytic stratification. In 4.5 we discuss how the slicing and intersection theories of [4] and [5] generalize to these maps and chains. For a subanalytic map

Received by the editors March 15, 1974.

AMS (MOS) subject classifications (1970). Primary 32B20, 57A60, 55B45; Secondary $32 \mathrm{C} 30,55 \mathrm{~B} 35$.

Key words and phrases. Stratification, semianalytic, subanalytic, subanalytic chain, slice, intersection.

(1) This work was partially supported by National Science Foundation grant GP29321. 
$f$ (which may fail to be even locally Lipschitzian), the chain map $f_{\#}$ is defined in 4.4. Use of this map in 4.6 where $f$ is a strong deformation retract obtained from a subanalytic CW decomposition offers a much easier treatment of the resulting subanalytic homology theory than the approach of [6] which treated the homology of semianalytic sets using chains defined by only semianalytic sets. In 4.7-4.8 we describe tangent cones and joins of subanalytic sets and chains as well as mapping cylinders and cones of subanalytic maps.

2. Preliminaries. For any subset $C$ of a topological space, the frontier of $C$ (denoted Fron $C$ ) is the set $(C l o s ~ C) \sim C$.

For any subset $D$ of a class 1 manifold, a stratification $\mathcal{S}$ of $D$ is a locally finite partition of $D$ into (properly embedded) class 1 submanifolds $S$ such that

$D \cap$ Fron $S=\bigcup S \cap\{R: R \cap \operatorname{Clos} S \neq \varnothing$ and $\operatorname{dim} R<\operatorname{dim} S\}$.

For any two families $\mathcal{E}$ and $\mathcal{F}$ of subsets of the same set, $\mathcal{E}$ is compatible with $\mathcal{F}$ if $E \subset F$ whenever $E \in \mathcal{E}, F \in \mathcal{F}$, and $E \cap F \neq \varnothing$.

A subset $A$ of a real analytic manifold $M$ is semianalytic if $M$ can be covered by open sets $U$ so that $U \cap A$ is a union of sets, each of which is a connected component of $g^{-1}\{0\} \sim b^{-1}\{0\}$ for $g$ and $b$ belonging to some finite family of real-valued functions analytic in $U$.

A subset $B$ of a real analytic manifold $N$ is subanalytic if $N$ can be covered by open sets $V$ so that $V \cap B$ is a union of sets, each of which is a connected component of $f(G) \sim f(H)$ for $G$ and $H$ belonging to some finite family $\varrho$ of semianalytic subsets of an analytic manifold $M$ and analytic mapping $f: M \rightarrow N$ whose restriction to Clos $\bigcup \mathcal{G}$ is proper.

It readily follows $[7,3.1]$ that the intersection, difference, locally finite union, and Cartesian product of semianalytic (resp., subanalytic) sets is semianalytic (resp., subanalytic) and that the connected component of or the inverse image under an analytic map of a semianalytic (resp., subanalytic) set is semianalytic (resp., subanalytic); moreover, the direct image of a subanalytic set $B$, under an analytic map whose restriction to Clos $B$ is proper, is also subanalytic.

A stratification of a subset of a real analytic manifold is called semianalytic (resp., subanalytic) if each of its members is a semianalytic (resp., subanalytic) real analytic submanifold.

The following main theorem which we use is established in [11, PP. 150153] for the semianalytic case and in $[7,4.2]$ or $[9,4.8]$ for the subanalytic case. 
Stratification Theorem. For any locally finite family $\mathbb{Q}$ of semianalytic (resp., subanalytic) subsets of a paracompact real analytic manifold $M$, there is a semianalytic (resp., subanalytic) stratification of $M$ compatible with $\mathcal{G}$.

For any semianalytic (resp., subanalytic) set $A$ and semianalytic (resp., subanalytic) stratification $\delta$ of $A$, the number

$$
\sup (\{-1\} \cup\{\operatorname{dim} S: S \in S\}) \in\{-1,0,1,2, \ldots\} \cup\{+\infty\}
$$

thus is independent of $\mathcal{S}$ and is called the dimension of $A$ and denoted $\operatorname{dim} A$. In case $A$ is nonempty, we also conclude that Fron $A$ is semianalytic (resp., subanalytic) with $\operatorname{dim}($ Fron $A) \leq(\operatorname{dim} A)-1$, and infer from $[7,4.2]$ or from $[7,2.3]$ as in the argument of [2, p. 336] that $\operatorname{dim} A \leq k$ if and only if $\mathcal{H}^{k}(A \cap K)<\infty$ for every compact $K$ where $\mathcal{H}^{k}$. denotes the $k$ dimensional Hausdorff measure $[2,2.10 .2(1)]$ corresponding to any Riemannian metric.

Our notations, with the exception of the groups $Z_{k}(A, B), \mathscr{B}_{k}(A, B)$, and $H_{k}(A, B)$ defined in 4.6, follow those of [2, pp. 669-671]. We also fix $n \in\{1,2, \ldots\}$ and let

$$
\begin{gathered}
I=\mathbf{R} \cap\{t: 0 \leq t \leq 1\}, \quad I^{0}=\mathbf{R}^{0}=\{0\}, \\
p_{0}: \mathbf{R}^{n} \rightarrow \mathbf{R}^{0}, \quad q_{1}: \mathbf{R}^{1} \rightarrow \mathbf{R}^{0}, \quad p_{0}(x)=0=q_{1}(t) \quad \text { for } x \in \mathbf{R}^{n} \text { and } t \in \mathbf{R}^{1}, \\
\quad p_{i}: \mathbf{R}^{n} \rightarrow \mathbf{R}^{i}, \quad q_{i+1}: \mathbf{R}^{i+1} \rightarrow \mathbf{R}^{i}, \\
p_{i}\left(x_{1}, \ldots, x_{n}\right)=\left(x_{1}, \ldots, x_{i}\right)=q_{i+1}\left(x_{1}, \ldots, x_{i+1}\right) \\
\text { for } i \in\{1,2, \ldots, n\} \text { and }\left(x_{1}, \ldots, x_{n+1}\right) \in \mathbf{R}^{n+1} .
\end{gathered}
$$

3. Stratifications and $\mathrm{CW}$ decompositions.

3.1. Theorem. Suppose $K$ is a compact subset of $\mathbf{R}^{n}, \delta_{0}=\left\{\mathbf{R}^{0}\right\}$, and for each $i \in\{1,2, \ldots, n\}, \delta_{i}$ is a stratification of $p_{i}(K)$ such that $\delta_{i-1}$ is compatible with $\left\{q_{i}(S): S \in \mathcal{S}_{i}\right\}$ and $D\left(q_{i} \mid S\right)(x)$ is injective whenever $x \in S \in \mathcal{S}_{i}$ and $\operatorname{dim} S<i$. If $\mathcal{C}_{0}=\left\{\mathbf{R}^{0}\right\}$ and, for each $i \in\{1,2, \cdots, n\}$, $\mathbb{Q}_{i}=\left\{A: A\right.$ is a component of $q_{i}^{-1}(C) \cap S, C \in \mathcal{C}_{i-1}, S \in \mathcal{S}_{i}$, and $\left.\operatorname{dim} S<i\right\}$, $B_{i}=\left\{B: B\right.$ is a component of $q_{i}^{-1}(C) \cap S, C \in \mathcal{C}_{i-1}, S \in \mathcal{S}_{i}$, and $\left.\operatorname{dim} S=i\right\}$, and $\mathcal{C}_{i}=\mathscr{Q}_{i} \cup \mathfrak{B}_{i}$, then the following five conclusions are true:

(1) Each point in $\mathbf{R}^{i}$ is the center of an arbitrarily small open rectangle whose intersection with every member of $\mathcal{C}_{i}$ is connected. 
(2) For each $A \in \mathbb{Q}_{i}, q_{i}(A) \in \mathcal{C}_{i-1}$ and $q_{i} \mid \operatorname{Clos} A$ is injective.

(3) For each $B \in \mathfrak{B}_{i}$, $(1-t) w+t x$ belongs to $B$ whenever $t \in I, w$ and $x$ belong to $B$, and $q_{i}(w)=q_{i}(x)$.

(4) For each $C \in \mathcal{C}_{i}$, the two sets,

$$
\begin{aligned}
& A_{C}^{-}=q_{i}^{-1}\left[q_{i}(C)\right] \cap\left\{\left(x_{1}, \ldots, x_{i}\right): x_{i}=\inf \left\{z:\left(x_{1}, \ldots, x_{i-1}, z\right) \in C\right\}\right\} \\
& A_{C}^{+}=q_{i}^{-1}\left[q_{i}(C)\right] \cap\left\{\left(x_{1}, \ldots, x_{i}\right): x_{i}=\sup \left\{z:\left(x_{1}, \ldots, x_{i-1}, z\right) \in C\right\}\right\}
\end{aligned}
$$

belong to $\mathfrak{Q}_{i}$. If $f_{\mathrm{R}^{0}}=0 \in \mathrm{R}^{0}$, and

$$
\begin{aligned}
& { }_{C_{C}}: I \times \operatorname{Clos} q_{i}(C) \rightarrow \mathrm{R}^{i}, \quad f_{C}: I^{i} \rightarrow \mathrm{R}^{i}, \\
& { }{ }_{C}(t, y)=(1-t)\left(q_{i} \mid \operatorname{Clos} A_{C}^{-}\right)^{-1}(y)+t\left(q_{i} \mid \operatorname{Clos} A_{C}^{+}\right)^{-1}(y), \\
& f_{C}\left(t_{1}, \ldots, t_{i}\right)=b_{C}\left[t_{i}, f_{q_{i}(C)}\left(t_{1}, \ldots, t_{i-1}\right)\right]
\end{aligned}
$$

for $(t, y) \in I \times \operatorname{Clos} q_{i}(C)$ and $\left(t_{1}, \cdots, t_{i}\right) \in I^{i}$, and

$$
I_{C}=I^{i} \cap\left\{\left(t_{1}, \ldots, t_{i}\right): t_{j}=0 \text { whenever } p_{j}\left[p_{i}^{-1}(C)\right] \in \mathbb{Q}_{j}\right\},
$$

then $\operatorname{dim} I_{C}=\operatorname{dim} C$, and $f_{C}$ maps $I_{C}$ continuously onto $C l o s C$ and $I_{C} \cap$ (Int $I)^{i}$ diffeomorphically onto $C$.

(5) $\mathcal{C}_{i}$ is a finite stratification and $C W$ decomposition $[1, \mathrm{~V}, 2.1]$ compatible with $\mathcal{S}_{i}$.

Proof. We use induction on $n$. Since $\delta_{1}$ consists of singleton sets and open intervals, the case $n=1$ is easily treated. By induction we assume conclusions (1) through (5) hold for $i \in\{1,2, \ldots, n-1\}$, and we abbreviate $q=q_{n}$

We will first prove that, for each $S \in \mathcal{S}_{n}$ with $\operatorname{dim} S<n$,

$$
j_{S}=\sup \left\{\operatorname{card}\left(q^{-1}\{y\} \cap S\right): y \in \mathrm{R}^{n-1}\right\}
$$

is finite. In fact, every $\operatorname{dim} S$ dimensional $C \in \mathcal{C}_{n-1}$ which intersects $q(S)$ is contained in $q(S) \sim q($ Fron $S)$ and is, by (4), diffeomorphic to the simplyconnected set $I_{C} \cap(\text { Int } I)^{i}$. Since $q\left|q^{-1}(C) \cap S=q\right| q^{-1}(C) \cap C l o s S$ is a covering map with compact fibers, $\mathfrak{Q}_{C}=\left\{A: A\right.$ is a component of $q^{-1}(C) \cap$ $S\}$ is a finite family each of whose members is mapped by $q$ diffeomorphically onto $C$. For any $y \in q(S)$ and finite subset $F$ of $q^{-1}\{y\} \cap S$, there are, by (1), an open neighborhood $V$ of $y$ and disjoint neighborhoods $U_{x}$ of each $x \in F$ such that $V \cap C$ is connected for all $C \in \mathcal{C}_{n-1}$ and $q$ maps $U_{x} \cap S$ diffeomorphically onto a closed submanifold of $V$. Since $\operatorname{dim} q\left(U_{x} \cap S\right)=$ 
$\operatorname{dim} S, U_{x} \cap A$ is nonempty for some $\operatorname{dim} S$ dimensional $C \in \mathcal{C}_{n-1}$ and $A \in \mathbb{Q}_{C}$. Inasmuch as $q\left(U_{x} \cap A\right) \cap C$ is open relative to $C, V \cap C \subset q\left(U_{x} \cap A\right)$, $U_{x} \cap A=\left(q \mid U_{x} \cap S\right)^{-1}(V \cap C)$, and $U_{w} \cap A=\varnothing$ whenever $x \neq w \in F$. Thus

card $F \leq \sum\left\{\right.$ ard $\mathbb{Q}_{C}: q(S) \supset C \in \mathcal{C}_{n-1}$ and $\left.\operatorname{dim} C=\operatorname{dim} S\right\}$.

To prove (2) for any $A \in \mathbb{Q}_{n}$, we observe that, for every $y \in C \in \mathcal{C}_{n-1}$ and $E \in \mathbb{Q}_{n}$, each $x \in q^{-1}\{y\} \cap E$ has a neighborhood whose intersection with $E$ is mapped diffeomorphically by $q$ onto a neighborhood of $y$ relative to $C$. Inasmuch as there are only finitely many such $x$ and $\mathbb{Q}_{n}$ is compatible with $\{$ Fron $A\}, q(A) \cap q($ Fron $A)=\varnothing$. Thus $q \mid A$ is a covering map, and $q(A) \in \mathcal{C}_{n-1}$. Since $q(A)$ is, by (4), simply-connected, $q \mid A$ is injective, and $A_{A}^{-}=A=A_{A}^{+}$.

Moreover, $q \mid \mathrm{Clos} A$ is injective. In fact, for any $y \in \operatorname{Clos} q(A)=$ $q(\operatorname{Clos} A)$, we may choose disjoint neighborhoods $W_{x}$ about each $x$ in the finite set $G=q^{-1}\{y\} \cap \operatorname{Clos} A$. The intersection with $q(A)$ of the neighborhood $\mathbf{R}^{n-1} \sim q\left[(\operatorname{Clos} A) \sim \bigcup_{x \in G} W_{x}\right]$ of $y$ then contains distinct components for every $x \in G$, each of whose closures contains $y$. From (1) applied to $q(A)$, we conclude that card $G=1$.

To establish (3) for any $B \in \mathscr{B}_{n}$ we use (2) to observe that $q(B) \epsilon$ $\mathcal{C}_{n-1}$ and that, for those $A \in \mathbb{Q}_{n}$ with $q(A)=q(B)$, the functions $g_{A}: q(A)$ $\rightarrow \mathbf{R}$ such that

$$
(q \mid A)^{-1}\left(y_{1}, \ldots, y_{n-1}\right)=\left(y_{1}, \ldots, y_{n-1}, g_{A}\left(y_{1}, \ldots, y_{n-1}\right)\right)
$$

whenever $\left(y_{1}, \ldots, y_{n-1}\right) \in q(A)$, are, by the intermediate value theorem, well-ordered by inequality; thus $q^{-1}[q(B)] \cap$ Fron $B$ is the union of precisely two such $A$, which are necessarily $A_{B}^{-}$and $A_{B}^{+}$.

To show (1) we assume $c=\left(c_{1}, \ldots, c_{n}\right) \in K, \mathcal{C}=\mathcal{C}_{n} \cap\{C: c \in C \operatorname{los} C\}$, $\epsilon>0$, and $\delta=\inf \{\epsilon, \operatorname{dist}(c, F$ ron $\cup \mathcal{C})\}$, and use (2) and induction to choose an open rectangle $Y$ centered at $q(c)$ in $\mathrm{R}^{n-1} \cap \mathrm{U}[q(c), \delta / \sqrt{2}]$ such that $Y$ intersects every member of $\mathcal{C}_{n-1}$ in a connected set and

$$
\operatorname{dist}\left(c, q^{-1}\{y\} \cap \operatorname{Clos} A\right)<\delta / \sqrt{2}
$$

whenever $A \in \mathbb{Q}_{i} \cap \mathcal{C}$ and $y \in Y \cap q(\operatorname{Clos} A)$. Then

$$
X=q^{-1}(Y) \cap\left\{\left(x_{1}, \ldots, x_{n}\right):\left|x_{n}-c_{n}\right|<\delta / \sqrt{2}\right\}
$$

is an open rectangle centered at $c$ in $\mathbf{R}^{n} \cap \mathrm{U}(c, \epsilon)$ whose intersection with any member of $\mathcal{C}_{n}$ is, by (2) and (3), connected.

To prove (4) for any $C \in \mathcal{C}_{n}$, we compute 


$$
\begin{aligned}
& \operatorname{dim} C=\operatorname{dim} q(C)=\operatorname{dim} I_{q(C)}=\operatorname{dim} I_{C} \text { in case } C \in \mathbb{P}_{n}, \\
& \operatorname{dim} C=1+\operatorname{dim} q(C)=1+\operatorname{dim} I_{q(C)}=\operatorname{dim} I_{C} \text { in case } C \in B_{n},
\end{aligned}
$$

and note that im $h_{C}=C \operatorname{los} C, h_{C}[($ Int $I) \times q(C)]=C$, and $(q \mid A)^{-1}$ is of class 1 whenever $A \in \mathbb{Q}_{n}$.

To obtain (5) we now need only observe that $\mathcal{C}_{n}$ is finite because of the estimates

$$
\begin{aligned}
& \operatorname{card} \mathbb{Q}_{n} \leq\left(\operatorname{card} \mathcal{C}_{n-1}\right) \cdot \sum\left\{j_{S}: S \in S_{n} \text { and } \operatorname{dim} S<n\right\}, \\
& \operatorname{card} \mathbb{B}_{n}=\operatorname{card} \mathbb{Q}_{n}+\operatorname{card} \mathcal{C}_{n-1} .
\end{aligned}
$$

3.2. Lemma. For any finite family $\mathfrak{Q}$ of bounded semianalytic (respectively, subanalytic) subsets of $\mathbf{R}^{n}$, there are an orthogonal projection $q: \mathbf{R}^{n} \rightarrow \mathbf{R}^{n-1}$ and semianalytic (resp., subanalytic) stratification $\mathcal{S}$ of Clos U氏 compatible with $\mathfrak{Q}$ such that, for each $S \in \mathcal{S}, q(S)$ is semianalytic (resp., subanalytic) and, in case $\operatorname{dim} S<n, D(q \mid S)(x)$ is injective for all $x \in S$.

Proof. Choose, by the stratification theorem, a semianalytic (resp., subanalytic) stratification $\mathcal{P}$ of Clos $\cup \mathfrak{Q}$ compatible with $\mathfrak{Q}$, and let $\mathcal{Q}=$ $\mathcal{P} \cap\{Q: \operatorname{dim} Q<n\}$.

In the semianalytic case, we may, by [6, 2.4], choose an orthogonal projection $q: \mathbf{R}^{n} \rightarrow \mathbf{R}^{n-1}$ so that $q^{-1}\{y\}$ is nonsingular for $\mathcal{Q}$ whenever $y \in \mathbf{R}^{n-1}$ and infer from $[6,2.2,2.7(3)]$ that $q(S)$ is semianalytic for any $S \in \mathcal{P} \sim \mathcal{Q}$ or any semianalytic subset $S$ of $\mathcal{U}$. In the subanalytic case, each $Q \in \mathcal{Q}$ admits a countable cover by open balls $U$ for which there is a not-identically-zero analytic function $f: U \rightarrow \mathbf{R}$ with $U \cap Q \subset f^{-1}$ \{0\}. Applying $[6,2.3]$ to all such $U$ and $f$ for all $Q \in 2$, we obtain an orthogonal projection $q: \mathbf{R}^{n} \rightarrow \mathbf{R}^{n-1}$ such that, in either case, $\operatorname{dim}\left(q^{-1}\{y\} \cap Q\right) \leq 0$ whenever $Q \in \mathcal{Q}$ and $y \in \mathbf{R}^{n-1}$. Since, by the argument of [4, 2.9] (resp., $[7,4.3]), Q \cap\{x: D(q \mid Q)(x)$ is not injective $\}$ is contained in a (dim $Q)-1$ dimensional semianalytic (resp., subanalytic) set, we may re-stratify U2, using downward induction as in the proof of $[7,2.1]$ to obtain a semianalytic (resp., subanalytic) stratification $R$ of $\mathcal{Q}$ compatible with 2 such that $D(q \mid R)(x)$ is injective whenever $x \in R \in R$. The lemma follows with $\delta=$ $(\mathcal{P} \sim 2) \cup R$.

3.3. Theorem. For any locally finite family $\mathfrak{Q}$ of semianalytic (resp., subanalytic) subsets of a paracompact real analytic manifold $M$, there are a semianalytic (resp., subanalytic) stratification $D$ of $M$ compatible with $\mathbb{Q}$ and, for each $D \in \mathfrak{D}$, a continuous function $f_{D}$ mapping $I^{\operatorname{dim} D}$ onto 
Clos $D$ whose restriction to Int $I^{\operatorname{dim} D}$ is an analytic isomorphism and whose graph is semianalytic (resp., subanalytic).

Proof. Choose, for $i \in\{1,2, \ldots\}$, a compact semianalytic set $K_{i}$ along with an analytic isomorphism $g_{i}$ mapping some neighborhood of $K_{i}$ into $\mathbf{R}^{\operatorname{dim} M}$ such that $\left\{\right.$ Int $K_{1}$, Int $\left.K_{2}, \ldots\right\}$ is a locally finite cover of $M$. Letting $K_{0}=\varnothing$ and $\mathscr{D}_{0}=\{\varnothing\}$ and having chosen $\mathscr{D}_{0}, \mathscr{D}_{1}, \ldots, \mathscr{D}_{i-1}$ and

$$
\mathfrak{Q}_{i}=\left\{A \cap \operatorname{Int} K_{i}: A \in \mathbb{Q}\right\} \cup\left\{D \cap \operatorname{Int} K_{i}: D \in \bigcup_{j=0}^{i-1} \mathscr{D}_{j}\right\},
$$

we apply 3.2 repeatedly with $n=\operatorname{dim} M,(\operatorname{dim} M)-1, \ldots, 1$ to choose an orthogonal transformation $g$ of $\mathbf{R}^{\operatorname{dim} M}$ so that the hypotheses of 3.1 hold with $n=\operatorname{dim} M, K=g\left[g_{i}\left(K_{i} \sim \bigcup_{j=0}^{i-1}\right.\right.$ Int $\left.\left.K_{j}\right)\right]$, and $\delta_{n}$ compatible with $\left\{g\left[g_{i}(A)\right]: A \in \mathbb{Q}_{i}\right\}$; from 3.1 we thus obtain a semianalytic (resp., subanalytic) stratification $\mathfrak{D}_{i}$ of $K_{t} \sim \bigcup_{j=0}^{i-1}$ Int $K_{j}$ compatible with $\mathfrak{Q}_{i}$ along with suitable functions $f_{D}$ for $D \in \mathscr{D}_{i}$. The theorem follows with $\mathfrak{D}=\bigcup_{i=1}^{\infty} \mathscr{D}_{i} \cap$ $\left\{D: D \subset\right.$ Int $\left.K_{i}\right\}$.

3.4. Corollary. If $A \supset B$ are semianalytic (resp., subanalytic) subsets of $M$, then there exist an open semianalytic (resp., subanalytic) neighborhood $U$ of $A$ in $M$ and a strong deformation retraction of $U \cap A$ onto $B$ whose graph is semianalytic (resp., subanalytic).

Proof. With $m \in\{1,2, \ldots\}, J=[R \cap\{t:|t| \leq 1\}]^{m} \sim\{0\}$, and $\left\|\left(x_{1}, \ldots, x_{m}\right)\right\|=\sup \left\{\left|x_{1}\right|, \ldots,\left|x_{m}\right|\right\}$ for $\left(x_{1}, \ldots, x_{m}\right) \in \mathbf{R}^{m}$, we observe that the set

$$
\begin{aligned}
& (I \times J \times J) \cap\{(t, x, y): y=(1-t) x+t(x /\|x\|)\} \\
& =\bigcup_{i=1}^{m}(I \times J \times J) \cap\left\{\left(t,\left(x_{1}, \ldots, x_{m}\right),\left(y_{1}, \ldots, y_{m}\right)\right):\right. \\
& x_{i}^{2} \geq x_{j}^{2},\left[y_{j}-(1-t) x_{j}\right] x_{j} \geq 0 \text { and } \\
& \left.\quad\left[y_{j}-(1-t) x_{j}\right]^{2} x_{i}^{2}=t^{2} x_{j}^{2} \text { for } j=1, \ldots, m\right\}
\end{aligned}
$$

is semianalytic and use 3.3 and the reasoning of $[1, \mathrm{~V}, 2.12,2.14(3)]$.

4. Subanalytic constructions.

4.1. Subanalytic maps. We will work in the subanalytic category where an object is a pair $(M, A)$ such that $M$ is a paracompact real analytic manifold and $A$ is a subanalytic subset of $M$,

a morphism (which we call a subanalytic map) from $(M, A)$ to $(N, C)$ is 
a continuous function $f: A \rightarrow C$ whose graph

$$
f=(A \times C) \cap\{(x, y): y=f(x)\}
$$

is a subanalytic subset of $M \times N$, and

composition has the usual meaning.

The inverse image under a subanalytic map or the direct image under a proper subanalytic map of a subanalytic set is subanalytic. Moreover, the union of any two subanalytic maps which agree on the intersection of their domains is a subanalytic map.

4.2. Subanalytic chains. For any paracompact real analytic manifold $M, k \in\{0,1,2, \ldots\}$, and linear map $T: \mathfrak{D}^{k}(M) \rightarrow \mathbf{R}$, we use the notations:

spt $T=M \sim \bigcup\{U: U$ is open and $T(\phi)=0$.

$$
\text { whenever } \left.\phi \in \mathscr{T}^{k}(U) \text { has spt } \phi \subset U\right\} \text {, }
$$

and, in case $k \geq 1$,

$$
\partial T: \mathfrak{D}^{k-1}(M) \rightarrow \mathbf{R}, \partial T(\psi)=T(d \dot{\psi}) \quad \text { for } \psi \in \mathfrak{D}^{k-1}(M) .
$$

We say $T$ is a $k$ dimensional subanalytic chain in $M$ if $T$ satisfies either of the following two conditions which are, by the argument of $[2,4.2 .28]$, equivalent.

(1) $T=\Sigma_{E \in \xi^{-}} m_{E}\left(\mathcal{H}^{k} L E\right) \wedge \xi_{E}$ where $\mathcal{E}$ is a family of $k$ dimensional strata belonging to some subanalytic stratification of $M$ and, for each $E \in \mathcal{E}$, $m_{E}$ is an integer, $E$ is orientable with orienting $k$ vectorfield $\xi_{E}$, and

$$
\left[\left(\mathcal{H}^{k} L E\right) \wedge \xi_{E}\right](\phi)=\int_{E}\left\langle\phi(x), \xi_{E}(x)\right\rangle d \mathcal{H}^{k} x \text { for } \phi \in \mathcal{D}^{k}(M) .
$$

(2) $T$ is a locally integral flat chain $[2,4.1 .24]$, spt $T$ is contained in some $k$ dimensional subanalytic set, and spt $\partial T$ is contained in some $k-1$ dimensional subanalytic set.

From (1) it follows that if $T \neq 0$, then spt $T=\bigcup\{C l o s E: E \in \mathcal{E}$ and $\left.m_{E} \neq 0\right\}$ is a $k$ dimensional subanalytic set. From (2) it follows that $\partial T$ is a $k-1$ dimensional subanalytic chain in case $k \geq 1$.

For any real analytic mapping $b$ of $M$ into a real analytic manifold $N$ such that $b \mid$ spt $T$ is proper, $b_{\#} T$, where $\left(b_{\#} T\right)(\phi)=T\left(b^{\#} \phi\right)$ for $\phi \in D^{k}(N)$, is a $k$ dimensional subanalytic chain in $N$ by (2) because $b_{A} T$ is a locally integral flat chain, spt $b_{\#} T \subset b($ spt $T)$ and spt $\partial\left(b_{\#} T\right)=\operatorname{spt} b_{H}(\partial T) \subset$ $b($ spt $\partial T)$. To extend this notion to subanalytic maps we will use the following lemma and, for $A \subset \dot{M}$, the notation $\mathcal{C}_{k}(A)=\{T: T$ is a $k$ dimensional subanalytic chain and spt $T \subset A\}$. 
4.3. Lifting Lemma. If $p$ is an analytic mapping of an open neighborhood of a subanalytic set $G$ such that $p \mid G$ is a homeomorphism, then $p_{\#}$ maps $\mathcal{C}_{k}(G)$ isomorphically onto $\mathcal{C}_{k}[p(G)]$.

Proof. Suppose $\mathscr{D}$ is a subanalytic stratification of $G, \mathcal{E}=\mathscr{D} \cap$ $\{E: \operatorname{dim} E=k\}$, and $p \mid E$ is an analytic isomorphism whenever $E \in \mathcal{D}$. Then for any orientable $E \in \mathcal{E}$, the mapping $\Pi_{E}$, for which

$$
\Pi_{E}(\xi)(y)=\left\langle\bigwedge_{k} D(p \mid E)(x), \xi(x)\right\rangle /\left|\left\langle\bigwedge_{k} D(p \mid E)(x), \xi(x)\right\rangle\right|,
$$

whenever $\xi$ is an orienting $k$ vectorfield of $E, y \in p(E)$, and $x=(p \mid E)^{-1}(y)$, is a bijection between the orienting $k$ vectorfields of $E$ and $p(E)$.

To prove surjectivity we assume $T \in \mathcal{C}_{k}[p(G)]$ and use the stratification theorem and $[7,4.3]$ to choose $D$ compatible with $\left\{p^{-1}\right.$ (spt $\left.T\right), p^{-1}$ (spt $\left.\left.\partial T\right)\right\}$. By the constancy theorem of $[2,4.1 .31(2)]$, there exist, for each $E \in \mathcal{E}$, an integer $r_{E}$ and an orienting $k$ vectorfield $\rho_{E}$ of $p(E)$ such that $p(E) \cap$

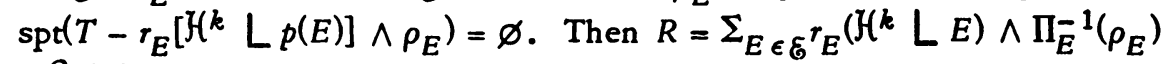
$\epsilon \mathcal{C}_{k}(G)$ and $p_{A} R-T$, having support in the at most $k-1$ dimensional subanalytic set $\bigcup\{p(D): D \in \mathcal{D} \sim \mathcal{E}\}$, equals zero by $4.2(1)$.

To prove injectivity we assume $Z \in \mathcal{C}_{k}(G)$ and $p_{\#} Z=0$ and use the stratification theorem and $[7,4.3]$ to choose $D$ compatible with $\{$ spt $Z$, spt $\partial Z\}$. By $[2,4.1 .31(2)]$, there exist, for each $E \in \mathcal{E}$, an integer $z_{E}$ and an orienting $k$ vectorfield $\zeta_{E}$ of $E$ such that $E \cap \operatorname{spt}\left[Z-z_{E}\left(\mathcal{H}^{k} L E\right) \wedge \zeta_{E}\right]$ $=\varnothing$; moreover $z_{E}=0$ because

$$
z_{E}\left[Y^{k} L_{p}(E)\right] \wedge \Pi_{E}\left(\zeta_{E}\right)=\left(p_{\#} Z\right) L_{p}(E)=0 .
$$

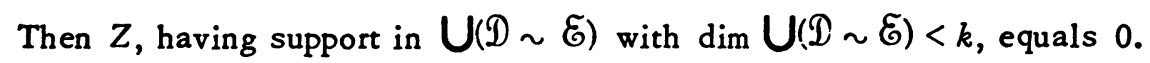

4.4. The chain homomorphism $f_{\sharp}$. Suppose $M$ and $N$ are paracompact real analytic manifolds, $p: M \times N \rightarrow M$ and $q: M \times N \rightarrow N$ are the projections, $A$ is a subanalytic subset of $M$, and $f: A \rightarrow N$ is a subanalytic map. Using 4.3, we define, for any $T \in \mathcal{C}_{k}(A)$ such that $f \mid$ spt $T$ is proper,

$$
f_{\#} T=q_{\#}\left[p_{\#} \mid \mathcal{C}_{k}(f)\right]^{-1}(T) \in \mathcal{C}_{k}(N),
$$

and verify the following four properties:

(1) $f_{\#}$ is a group homomorphism.

(2) $\operatorname{spt} f_{\#} T \subset q\left[f \cap p^{-1}(\mathrm{spt} T)\right]=f(\mathrm{spt} T)$.

$$
\begin{aligned}
\partial\left(f_{\#} T\right) & =q_{\#} \partial\left[p_{\#} \mid C_{k}(f)\right]^{-1}(T)=q_{\#}\left[p_{\#} \mid C_{k-1}(f)\right]^{-1} p_{\#} \partial\left[p_{\#} \mid C_{k}(f)\right]^{-1}(T) \\
& =q_{\#}\left[p_{\#} \mid C_{k-1}(f)\right]^{-1}(\partial T)=f_{\#}(\partial T)
\end{aligned}
$$

in case $k \geq 1$. 
(4) If $P$ is a paracompact real analytic manifold, $g: f(A) \rightarrow P$ is a subanalytic map, and $(g \circ f) \mid$ spt $T$ is proper, then $\left(g \circ f_{\#} T=g_{\sharp}\left(f_{\#} T\right)\right.$.

In fact, this is readily verified using the subanalytic set

$$
h=(u \times N \times P) \cap\{(x, y, z): y=f(x) \text { and } z=\delta(y)\},
$$

the projections

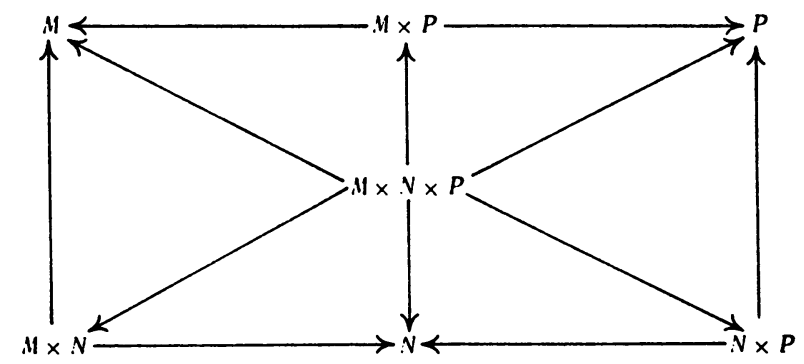

and 4.3 to chase the induced diagram

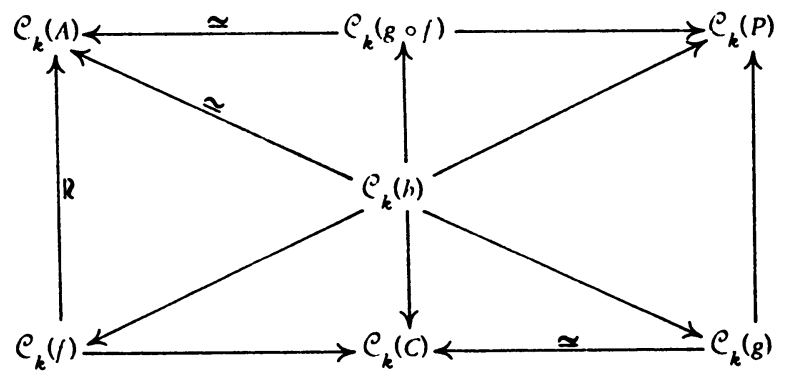

4.5. Slicing and intersection. Thanks to the bounds provided by $[7$, $4.2,4.3,2.3]$ the reasoning of $[4,2.9, \$ \$ 4-5]$ carries over to give an analogous slicing and intersection theory for subanalytic chains and real analytic maps.

In particular, for a $j$ dimensional subanalytic chain $R$ and a $k$ dimensional subanalytic chain $T$ in an oriented, paracompact real analytic manifold $M$, which intersect suitably in the sense that $j+k \geq \operatorname{dim} M$,

$$
\operatorname{dim}[(\operatorname{spt} R) \cap(\operatorname{spt} T)] \leq j+k-\operatorname{dim} M, \quad \text { and }
$$

$\operatorname{dim}[(\operatorname{spt} R \cap \operatorname{spt} \partial T) \cup(\operatorname{spt} \partial R \cap \operatorname{spt} T)] \leq j+k-1-\operatorname{dim} M$, a $j+k-\operatorname{dim} M$ dimensional subanalytic chain, $R \cap T$, is well-defined in $[4,5(1)]$ by slicing at 0 , in any coordinate neighborhood, $R \times T$, by the subtraction map. Subanalytic intersection theory satisfies the analogue of $[4,5.8]$ and is uniquely characterized by axioms corresponding to $[4,5.11]$.

To extend the slicing theory of $[4, \S 4]$ to subanalytic maps, we let 
$p: M \times \mathbf{R}^{n} \rightarrow M$ and $q: M \times \mathbf{R}^{n} \rightarrow \mathbf{R}^{n}$ be the projections and use 4.3, 4.4, and the subanalytic version of $[4,4.3]$ to define, for any $k$ dimensional subanalytic chain $T$ in $M$, any subanalytic map $f:$ spt $T \rightarrow \mathbf{R}^{n}$, and any $y \in \mathbf{R}^{n}$ with $\operatorname{dim}\left(f^{-1}\{y\} \cap\right.$ spt $\left.T\right) \leq k-n$ and $\operatorname{dim}\left(f^{-1}\{y\} \cap\right.$ spt $\left.\partial T\right) \leq k-n-1$, a $k-n$ dimensional subanalytic chain, the slice of $T$ in $f^{-1}\{y\}$,

$$
\langle T, f, y\rangle=p_{\#}\left\langle\left[p_{\#} \mid \mathcal{C}_{k}(f)\right]^{-1}(T), q, y\right\rangle,
$$

and infer that the function which associates $\langle T, f, y\rangle$ with such $y$ is continuous in the locally integral flat topology [2, 4.3.16].

The two statements,

$$
\begin{gathered}
\operatorname{spt}\langle T, f, y\rangle \subset f^{-1}\{y\} \cap \text { spt } T, \\
\partial\langle T, f, y\rangle=(-1)^{n}\langle\partial T, f, y\rangle \text { in case } k>n,
\end{gathered}
$$

readily follow from $[4,3.5(1)(2)]$. To obtain a formula corresponding to [4, 4.4] where $T, f$, and $g$ all are subanalytic, we use 4.3, the diagrams of 4.4 with $P=R^{l}$, and the subanalytic version of $[4,4.4]$ in case $f$ and $g$ are certain projections. A version. of $[4,4.5]$ where $S, f$, and $g$ are subanalytic is proven similarly. The proof of $[4,4.7]$ then carries over to the case where $b$ and $T$ are subanalytic.

For any integer $\nu \geq 2$, the results of [5] also generalize to subanalytic maps and the modulo $\nu$ congruence classes of subanalytic chains.

4.6. Subanalytic homology theory. Although it follows from 3.3 and the reasoning of $[1, \mathrm{~V}, \$ 4]$ that any two homology theories on the category of pairs of subanalytic sets and continuous maps between such pairs have isomorphic homology groups, the following subanalytic theory seems the most natural.

For $k \in\{0,1,2, \ldots\}$ and subanalytic sets $A \supset B$ in a real analytic manifold $M$, we define the group of subanalytic cycles,

$$
\begin{array}{r}
\mathcal{Z}_{k}(A, B)=\{T: T \text { is a subanalytic chain of compact support, } \\
\text { spt } T \subset A \text { and spt } \partial T \subset B \text { or } k=0\},
\end{array}
$$

the subgroup of subanalytic boundaries,

$$
\mathbb{B}_{k}(A, B)=\left\{R+\partial S: R \in \mathcal{Z}_{k}(B, B) \text { and } S \in \mathscr{Z}_{k+1}(A, A)\right\} \text {, }
$$

and the subanalytic bomology group,

$$
\mathbf{H}_{k}(A, B)=\mathscr{Z}_{k}(A, B) / \mathscr{B}_{k}(A, B) \text {. }
$$

For any subanalytic sets $C \supset D$ in a real analytic manifold $N$ and subanaly- 
tic map $f: A \rightarrow N$ with $f(A) \subset C$ and $f(B) \subset D, 4.4(1)(2)(3)$ imply that the map $f_{\#}: Z_{k}(A, B) \rightarrow Z_{k}(C, D)$ induces a homomorphism $\mathbf{H}_{k}(f): \mathbf{H}_{k}(A, B) \rightarrow$ $\mathbf{H}_{k}(C, D)$.

Repeating the argument of $[2,4.4 .1]$ gives a short proof of the validity of the Eilenberg-Steenrod axioms for the category of pairs of subanalytic sets and subanalytic maps between such pairs.

From 3.4 we readily infer that there exists an arbitrarily small subanalytic neighborhood $W$ of $B$ such that $\mathbf{H}_{k}(W \cap A, B) \simeq 0$ for all $k$. In particular there exist arbitrarily small open subanalytic neighborhoods $U$ of $A$ in $M$ with $H_{k}(U, A) \simeq 0$ and $V$ of $B$ in $U$ with $H_{k}(V, B) \simeq 0$ for all $k$; hence the inclusion map of $\mathscr{Z}_{k}(A, B)$ into $\mathscr{Z}_{k}(U, V)$ induces an isomorphism $\Phi_{k}$ : $\mathbf{H}_{k}(A, B) \simeq \mathbf{H}_{k}(U, V)$ for all $k$.

As in $[6,5.5]$ this allows us to define, by analytic (or subanalytic) approximation, $\mathbf{H}_{k}(f)$ for any continuous map $f$ between pairs of subanalytic sets and obtain a homology theory on the extended category.

Moreover, as in [6, 6.4], we have, for any orientation on $M$ and nonnegative integers $j, k$ with $j+k \geq \operatorname{dim} M$, an intersection product

$$
\text { ก: } \mathbf{I}_{j}(A, B) \times \mathbf{H}_{k}(A, B) \rightarrow \mathbf{H}_{j+k-\operatorname{dim} M}(A, B)
$$

which is well-defined, for $\rho \in \mathbf{H}_{i}(A, B)$ and $r \in \mathbf{H}_{k}(A, B)$, by

$$
\rho \cap \tau=\Phi_{j+k-\operatorname{dim} M}^{-1}\left[(R \cap T)+\mathscr{B}_{j+k-\operatorname{dim} M}(U, V)\right],
$$

where $R \in \Phi_{j}(\rho)$ and $T \in \Phi_{k}(\gamma)$ are chosen, using the subanalytic analogue of $[6,6.3]$, to intersect suitably. In case $M=R^{n}, R$ is a countable family of subanalytic chains of dimension at least $n-k$, and $Q \in \Phi_{k}(r)$, then simply the translation of $Q$ by almost all $y \in \mathrm{R}^{n}$ with

$$
|y|<\inf \left\{\operatorname{dist}\left(\operatorname{spt} Q, \mathbf{R}^{n} \sim V\right), \operatorname{dist}\left(\operatorname{spt} \partial Q, \mathbf{R}^{n} \sim V\right)\right\}
$$

belongs to $\Phi_{k}(\tau)$ and intersects suitably with every $R \in R$. Various properties of the homology intersection product corresponding to $[4,5.11]$ readily follow from the subanalytic analogues of $[4,5.11]$ and $[6,6.4]$.

4.7. Tangent cones. If $A$ is a semianalytic (resp., subanalytic) subset of $\mathbf{R}^{n}$ and $a \in \mathbf{R}^{n}$, then $\operatorname{Tan}(A, a)[2,3.1 .21]$ is semianalytic (resp., subanalytic) and $\operatorname{dim} \operatorname{Tan}(A, a) \leq \operatorname{dim} A$.

In fact if $a \notin \operatorname{Clos} A$, then $\operatorname{Tan}(A, a)=\varnothing$ or if $a \in(\operatorname{Clos} A) \sim$ $\operatorname{Clos}(A \sim\{a\})$, then $\operatorname{Tan}(A, a)=\{0\}$ and $\operatorname{dim} A \geq 0$. We now assume $a \epsilon$ $\operatorname{Clos}(A \sim\{a\})$; hence $\operatorname{Tan}(A, a)=\operatorname{Tan}(A \sim\{a\}, a)$ and let

$$
p:\left(\mathbf{R}^{n}\right)^{3} \rightarrow \mathbf{R}^{n}, \quad q:\left(\mathbf{R}^{n}\right)^{3} \rightarrow \mathbf{R}^{n}, \quad r:\left(\mathbf{R}^{n}\right)^{3} \rightarrow \mathbf{R}^{n}, \quad s:\left(\mathbf{R}^{n}\right)^{3} \rightarrow \mathbb{R}^{n},
$$


$p(x, y, z)=x, \quad q(x, y, z)=y, \quad r(x, y, z)=z, \quad$ and $\quad s(x, y, z)=x-y$,

for $(x, y, z) \in\left(\mathbf{R}^{n}\right)^{3}$. Then $r \mid s^{-1}\{0\} \cap p^{-1}\{a\}$ is an isomorphism, the secant space $[12, \S 4]$

$$
\begin{aligned}
L=\left(\mathbb{R}^{n}\right)^{3} \cap & \left\{\left(x_{1}, \ldots, x_{n}\right),\left(y_{1}, \ldots, y_{n}\right),\left(z_{1}, \ldots, z_{n}\right):\right. \\
& \left.\left(x_{i}-y_{i}\right) z_{i} \geq 0,\left(x_{i}-y_{i}\right) z_{j}=\left(x_{j}-y_{j}\right) z_{i} \text { for } i, j=1, \ldots, n\right\}
\end{aligned}
$$

is semianalytic, and thus

$$
\operatorname{Tan}(A, a)=r\left(s^{-1}\{0\} \cap \operatorname{Fron}\left[\left(L \sim s^{-1}\{0\}\right) \cap p^{-1}\{a\} \cap q^{-1}(A)\right]\right)
$$

is semianalytic (resp., subanalytic). Moreover $B=\left(L \sim s^{-1}\{0\}\right) \cap p^{-1}\{a\} \cap$ $q^{-1}(A)$ has dimension at most $(\operatorname{dim} A)+1$ because, for each $i \in\{1,2$, $\cdots, n\}$, the restriction of the projection

$$
\begin{aligned}
\pi_{i}:\left(\mathbf{R}^{n}\right)^{3} \rightarrow \mathbf{R}^{n} \times \mathbf{R}, \pi_{i}\left(x, y,\left(z_{1}, \ldots, z_{n}\right)\right)=\left(y, z_{i}\right) & \\
& \quad \text { for }\left(x, y,\left(z_{1}, \ldots, z_{n}\right)\right) \in\left(\mathbf{R}^{n}\right)^{3},
\end{aligned}
$$

maps the subanalytic set

$$
B \cap\left\{\left(\left(x_{1}, \ldots, x_{n}\right),\left(y_{1}, \ldots, y_{n}\right), z\right): x_{i} \neq y_{i}\right\}
$$

injectively into $A \times \mathbf{R}$. Thus $\operatorname{dim} \operatorname{Tan}(A, a) \leq \operatorname{dim}$ Fron $B \leq \operatorname{dim} A$. (Another proof of this inequality for the semianalytic case is given in [2, 3.4.11].)

Using the argument of $[2,4.3 .18]$, it then follows that for every $k$. dimensional subanalytic chain $T$ in $\mathbf{R}^{n}$ and a $\in \mathbf{R}^{n}$, the subanalytic chains $\left(\mu_{t} \circ r_{-a}\right)_{H} T$ converge in the locally integral flat topology as $t \rightarrow+\infty$ to a subanalytic chain.

4.8. Joins, mapping cyclinder, mapping cone. If $A$ and $B$ are subanalytic subsets of $\mathbf{R}^{m}$ and $F: \mathbf{R}^{m} \times \mathbf{R} \times \mathbf{R}^{m} \rightarrow \mathbf{R}^{m}, F(x, t, y)=(1-t) x+$ ty for $(x, t, y) \in \mathbf{R}^{m} \times \mathbf{R} \times \mathbf{R}^{m}$, then the join of the sets $A$ and $B$, $F(A \times I \times B)$, is an at most $(\operatorname{dim} A)+1+(\operatorname{dim} B)$ dimensional subanalytic set. If $S$ and $T$ are subanalytic chains, then the join of $S$ and $T[2,4.1 .11]$, $F_{\#}(S \times[0,1] \times T)$, is a $(\operatorname{dim} A)+1+(\operatorname{dim} B)$ dimensional subanalytic chain.

If $f: A \rightarrow \mathbf{R}^{n}$ is a subanalytic map, and

$$
\begin{gathered}
g: A \times l \rightarrow \mathbb{R}^{m} \times \mathbb{R}^{n} \times l, \quad h: \mathbf{R}^{m} \times \mathbb{R}^{n} \times l \rightarrow \mathbf{R}^{m} \times \mathbf{R}^{n} \times I, \\
g(x, t)=((1-t) x, \quad t f(x), t) \text { for }(x, t) \in A \times I, \\
h(x, y, t)=(2 t x, 2 t y, t) \quad \text { for } 0 \leq t \leq 1 / 2 \text { and }(x, y) \in \mathbb{R}^{m} \times \mathbb{R}^{n},
\end{gathered}
$$




$$
h(x, y, t)=(x, y, t) \text { for } 1 / 2 \leq t \leq 1 \text { and }(x, y) \in \mathbb{R}^{m} \times \mathbb{R}^{n} \text {, }
$$

then the mapping cyclinder of $f$, im $g$, and the mapping cone of $f$, im $(b \circ g)$, are $(\operatorname{dim} A)+1$ dimensional subanalytic subsets of $\mathbf{R}^{m} \times \mathbf{R}^{n} \times I$.

\section{BIBLIOGRAPHY}

1. A. Dold, Lectures on algebraic topology, Springer-Verlag, Berlin and New York, 1973.

2. H. Federer, Geometric measure theory, Die Grundlehren der math. Wissenschaften, Band 153, Springer-Verlag, New York, 1969. MR 41 \#1976.

3. B. Giesecke, Simpliziale Zerlegung abzählbarer analytischer Räume, Math. Z. 83 (1964), 177-213. MR $28 \# 2563$.

4. R. Hardt; Slicing and intersection theory for chains associated with real analytic varieties, Acta Math. 129 (1972), 57-136. MR 47 \#110.

5. Slicing and intersection theory for chains modulo $v$ associated with real analytic varieties, Trans. Amer. Math. Soc. 185 (1973), 327-340.

6. - Homology theory for real analytic and semianalytic sets, Ann. Scuola Norm. Sup. Pisa (4) 2 (1975).

7. - Stratification of real analytic mappings and images, Inventiones Math. 28 (1975), 193-208.

8. - Sullivan's local Euler characteristic theorem, Manuscripta Math. 12 (1974), 87-92.

9. H. Hironaka, Subanalytic sets, Number Theory, Algebraic Geometry, and Commutative Algebra, in Honor of Y. Akizuki, Kinokuniya, Tokyo, 1973, pp. 453-493.

10. S. Xojasiewicz, Triangulation of semi-analytic sets, Ann. Scuola Norm. Sup. Pisa (3) 18 (1964), 449-474. MR 30 \#3478.

11. - Ensembles semianalytiques, Cours Faculté des Sciences d'Orsay, Inst. Hautes Études Sci. Bures-sur-Yvette, 1965.

12. W. F. Pohl, Some integral formulas for space curves and their generalization, Amer. J. Math. 90 (1968), 1321-1345. MR 38 \#6523.

13. R. Hardt, Triangulation of subanalytic sets and proper light subanalytic maps (preprint).

SCHOOL OF MATHEMATICS, UNIVERSITY OF MINNESOTA, MINNEAPOLIS, MINNESOTA 55455 